\title{
ENERGY-PRESERVING INTEGRATORS FOR STOCHASTIC POISSON SYSTEMS*
}

\author{
DAVID COHEN ${ }^{\dagger}$ AND GUILLAUME DUJARDIN ${ }^{\ddagger}$
}

\begin{abstract}
A new class of energy-preserving numerical schemes for stochastic Hamiltonian systems with non-canonical structure matrix (in the Stratonovich sense) is proposed. These numerical integrators are of mean-square order one and also preserve quadratic Casimir functions. In the deterministic setting, our schemes reduce to methods proposed in [E. Hairer, JNAIAM. J. Numer. Anal. Ind. Appl. Math., 5(1-2), 73-84, 2011] and [D. Cohen, and E. Hairer, BIT, 51(1), 91-101, 2011].
\end{abstract}

Key words. Stochastic Poisson systems, Stratonovich SDEs, energy-preserving numerical schemes, stochastic midpoint scheme, Casimir function.

AMS subject classifications. 65C20, 65C30, 60H10, 60H35.

\section{Introduction}

We consider numerical discretisations of stochastic perturbations of Poisson systems of the form

$$
\mathrm{d} y_{t}=B\left(y_{t}\right) \nabla H\left(y_{t}\right)\left(\mathrm{d} t+c \circ \mathrm{d} W_{t}\right) .
$$

The above stochastic differential system is understood in the Stratonovich setting and the symbol o stands for the Stratonovich product. Here, $c \geq 0$ measures the size of the perturbation and $W_{t}$ is a one-dimensional Wiener process. Moreover, we assume that $B(y) \in \mathbb{R}^{d \times d}$ is a smooth skew-symmetric matrix-valued function and the Hamiltonian $H(y)$ is a sufficiently smooth scalar function of $y \in \mathbb{R}^{d}$ such that an exact solution of our problem exists for all time. This system describes a Hamiltonian motion perturbed by a multiplicative white noise which in some sense respects the geometric structure of the phase space; the randomness in the Hamiltonian vector field consists in a random force in the direction of the deterministic force and a random modification of the deterministic velocities that do not modify the structure of the phase space.

Stochastic canonical Hamiltonian systems (see for example [17] and references therein) of the form

$$
\mathrm{d} y_{t}=J^{-1} \nabla H\left(y_{t}\right)\left(\mathrm{d} t+\operatorname{cod} W_{t}\right)
$$

with $J:=\left(\begin{array}{cc}0 & I d \\ -I d & 0\end{array}\right)$, where $I d$ denotes an identity matrix, can be put into the form (1.1) by taking the constant matrix $B(y)=J^{-1}$. A typical example where the matrix in (1.1) is non-constant is given by a randomly perturbed rigid body problem [14, 13 , 1]; see also Section 4.2. That is, the motion of a rigid body in $\mathbb{R}^{3}$ subject to a white noise perturbation.

${ }^{*}$ Received: February 23, 2013; accepted (in revised form): October 29, 2013. Communicated by Weinan E.

${ }^{\dagger}$ Matematik och Matematisk Statistik, Umeå universitet, 90187 Umeå, Sweden (david.cohen@ math.umu.se).

${ }^{\ddagger}$ Inria Lille Nord-Europe and Laboratoire Paul Painlevé UMR CNRS 8524, 59650 Villeneuve d'Ascq Cedex, France (guillaume.dujardin@inria.fr). 


\section{ENERGY-PRESERVING INTEGRATORS FOR STOCHASTIC POISSON SYSTEMS}

An application of the chain rule for Stratonovich differential equations shows us immediately that the Hamiltonian $H(y)$ is a conserved quantity [18, 19, 20, 21, 26], that is

$$
H\left(y_{t}\right)=H\left(y_{0}\right) \equiv \text { Const },
$$

for all positive time $t$ along almost all realisations of the exact solution of (1.1). Here, $y_{0}$ denotes the initial value of the problem (1.1).

Recently, many efforts have been made to construct and analyse symplectic numerical schemes for stochastic Hamiltonian problems [17, 28, 3, 27, 22, 4, 15. In general, these schemes do not preserve exactly the Hamiltonian function $H(y)$. Our main interest in the present paper is thus the design of numerical integrators that exactly preserve the Hamiltonian $H(y)$. Let us mention that there is a lot of ongoing research activities in energy-preserving numerical integrators for deterministic problems, and various methods have been proposed in the literature; see [8, 25, 5, 9, 6]. However, in the stochastic setting, we are only aware of the numerical schemes proposed in [21] and in [7 for stochastic canonical Hamiltonian problems. Furthermore, [10] proposes a stochastic discrete gradient scheme which shares similarities with the numerical methods that we propose here (see below for more details). Closely related to the thematic addressed in these papers are the semianalytic numerical algorithms proposed in 23]; these splitting-step methods guarantee that the numerical solutions remain in a domain.

The present article is devoted to a stochastic extension of the (deterministic) methods introduced in [9] and in [6]. As we will show, our new numerical schemes exactly preserve the energy $H(y)$, quadratic Casimir functions, and are of mean-square order of convergence one. Moreover, they reduce to the method proposed in [21] and to one of the method proposed in [10] in the following canonical case:

$$
\mathrm{d} y_{t}=\left(\begin{array}{cc}
0 & -1 \\
1 & 0
\end{array}\right) \nabla H\left(y_{t}\right)\left(\mathrm{d} t+c \circ \mathrm{d} W_{t}\right)
$$

where $y_{t} \in \mathbb{R}^{2}, c$ is a real non-negative parameter, $W_{t}$ is a one-dimensional standard Wiener process, and $H(y)$ is a quadratic function of $y$. Let us also note that, again if $H(y)$ is a quadratic function, our numerical method reduces to the stochastic midpoint scheme from [17.

The new class of numerical schemes is presented in Section 2 and the main properties of the methods are proved in Section 3 . Finally, numerical experiments demonstrate the efficiency of our approach in Section 4 and possible extensions of this work are highlighted in the conclusion.

To close this introduction, let us mention several straightforward extensions of problems of the form (1.1) or (1.2) that one could discretise with similar techniques. Everything that is done in the present paper for one-dimensional white noise can easily be extended to the case

$$
\mathrm{d} y_{t}=B\left(y_{t}\right) \nabla H\left(y_{t}\right)\left(\mathrm{d} t+\sum_{j=1}^{m} c_{j} \circ \mathrm{d} W_{t}^{j}\right)
$$

where for all $j, c_{j} \geq 0$. One can also handle problems of the form

$$
\mathrm{d} y_{t}=B\left(y_{t}\right) \nabla H\left(y_{t}\right) \mathrm{d} t+\sum_{j=1}^{m} G^{j} \nabla H\left(y_{t}\right) \circ \mathrm{d} W_{t}^{j},
$$


with several skew-symmetric matrices $G^{j}$ of a special kind (see 7 for more details on the canonical Hamiltonian case $B(y)=J^{-1}$ ), and derive energy-preserving numerical schemes for these problems too. Closely related to the previous class of problems are the randomised Hamiltonian systems from [2, Chap. V.4], [20, and [12, Sect. 3.1]:

$$
\mathrm{d} y_{t}=J^{-1} \nabla H_{0}\left(y_{t}\right) \mathrm{d} t+\sum_{j=1}^{m} J^{-1} \nabla H_{j}\left(y_{t}\right) \circ \mathrm{d} W_{t}^{j},
$$

for which one can also derive numerical methods that preserve the first integral $H_{0}$ of the above problem. We recall, that $H$ is a first integral if one has $\left\{H, H_{0}\right\}=\left\{H, H_{1}\right\}=$ $\ldots=\left\{H, H_{m}\right\}=0$ with the Poisson bracket $\{\cdot, \cdot\}$ associated to the above problem. An extension of the proposed numerical integrators for the exact preservation of other (or multiple) first integrals is however not obvious.

\section{Energy-preserving schemes}

The proposed numerical scheme for the discretisation of (1.1) reads

$$
y_{n+1}=y_{n}+B\left(\frac{y_{n}+y_{n+1}}{2}\right) \int_{0}^{1} \nabla H\left(y_{n}+\tau\left(y_{n+1}-y_{n}\right)\right) \mathrm{d} \tau\left(h+c \Delta W_{n}\right),
$$

where $h$ denotes the time step and $\Delta W_{n}$ are Wiener increments.

One immediately sees that the above numerical scheme reduces to that of order two proposed in [6] in the deterministic context $(c=0)$. In fact, scheme (2.1) corresponds to that of [6] with a random step. Moreover, one notes that, if the matrix $B(y)=B$ is constant (e.g., if (1.1) is a canonical Hamiltonian problem as in [21, 17]) the method reduces to

$$
y_{n+1}=y_{n}+B \int_{0}^{1} \nabla H\left(y_{n}+\tau\left(y_{n+1}-y_{n}\right)\right) \mathrm{d} \tau\left(h+c \Delta W_{n}\right) .
$$

This is a natural extension of the second order scheme from [9] (see also the averaged vector field method from [25]) to the stochastic setting. We remark that another possibility would be to use the (deterministic) scheme proposed by Gonzalez [8] which would result in the symmetric discrete gradient method from [7. Additionally, as already noted in the introduction, when applied to (1.3), the numerical integrator (2.2) reduces to the scheme proposed in 21]; see Section 4 for more details.

REMARK 2.1. Because the proposed numerical methods are implicit with respect to both the drift and diffusion terms, some difficulties (essentially due to the unboundedness of $\Delta W_{n}$ ) may happen when implementing the schemes. As proposed by [17, one way to address this issue is to consider truncated random variables instead of the Wiener increments $\Delta W_{n}:=\sqrt{h} \xi$, with $\xi$ an $\mathcal{N}(0,1)$-distributed random variable. Indeed, setting $A_{h}:=\sqrt{2 k|\ln (h)|}$ (for an integer $k \geq 0$ ), one defines the truncated random variable [17]

$$
\zeta_{h}=\left\{\begin{array}{lll}
\xi, & \text { if } \quad|\xi| \leq A_{h} \\
A_{h}, & \text { if } \quad \xi>A_{h} \\
-A_{h}, & \text { if } \quad \xi<-A_{h}
\end{array}\right.
$$

Moreover, one has the following approximation property (see Lemma 2.1 from [17]):

$$
0 \leq \mathbb{E}\left[\xi^{2}-\zeta_{h}^{2}\right]=(1+2 \sqrt{2 k|\ln (h)|}) h^{k} .
$$


This is what we do in the present paper using the notation $\Delta \widehat{W}_{n}:=\sqrt{h} \zeta_{h}$ for the truncated random Wiener processes and taking $k=2$ in the definition of $A_{h}$. With this choice of truncated random variables, one can show that the stochastic midpoint scheme has order one [17, Th. 2.6] (this fact will be used in Theorem 3.3 below).

\section{Properties of the energy-preserving schemes}

The methods of the previous section have been designed to exactly preserve the Hamiltonian $H(y)$. It turns out that they enjoy further interesting properties. The proofs of the geometric properties of the numerical integrators follow easily from the one given in [6].

\subsection{Exact energy preservation.}

Proposition 3.1. If $B(y)$ is skew-symmetric for all $y$, then the numerical method (2.1) exactly preserves the energy, i.e., $H\left(y_{n}\right)=$ Const for all $n$.

Proof. From the fundamental theorem of calculus we have

$$
H\left(y_{n+1}\right)-H\left(y_{n}\right)=\int_{0}^{1} \nabla H\left(y_{n}+\tau\left(y_{n+1}-y_{n}\right)\right)^{\mathbf{T}}\left(y_{n+1}-y_{n}\right) \mathrm{d} \tau .
$$

From the definition of the method (2.1) we thus get for the difference above

$$
\left(\int_{0}^{1} \nabla H\left(y_{n}+\tau\left(y_{n+1}-y_{n}\right)\right) \mathrm{d} \tau\right)^{\mathbf{T}} B\left(\frac{y_{n}+y_{n+1}}{2}\right) \int_{0}^{1} \nabla H\left(y_{n}+\tau\left(y_{n+1}-y_{n}\right)\right) \mathrm{d} \tau\left(h+c \Delta \widehat{W}_{n}\right),
$$

which vanishes by the skew-symmetry of the matrix $B(y)$.

3.2. Conservation of quadratic Casimir's. A function $C(y)$ is called a Casimir function of the stochastic differential equation (1.1) if $\nabla C(y)^{\mathbf{T}} B(y)=$ 0 for all $y$. Along solutions of (1.1) we have $C\left(y_{t}\right)=$ Const, because $\mathrm{d} C\left(y_{t}\right)=$ $\nabla C\left(y_{t}\right)^{\mathbf{T}} B\left(y_{t}\right) \nabla H\left(y_{t}\right)\left(\mathrm{d} t+c \circ \mathrm{d} W_{t}\right)=0$. This property is independent of the Hamiltonian $H(y)$.

Proposition 3.2. Let $C(y)=y^{\mathbf{T}} A y$ (with a symmetric constant matrix $A$ ) be a Casimir function of the problem (1.1). The energy-preserving method (2.1) exactly preserves this Casimir.

Proof. Using again the fundamental theorem of calculus we have

$$
C\left(y_{n+1}\right)-C\left(y_{n}\right)=\int_{0}^{1} \nabla C\left(y_{n}+\tau\left(y_{n+1}-y_{n}\right)\right)^{\mathbf{T}}\left(y_{n+1}-y_{n}\right) \mathrm{d} \tau
$$

Because the integrand is a polynomial of degree 1 in $\tau$, an application of the midpoint quadrature rule gives the exact result. For the difference $C\left(y_{n+1}\right)-C\left(y_{n}\right)$, using the definition of the numerical scheme, we thus obtain

$$
\nabla C\left(y_{n}+\frac{1}{2}\left(y_{n+1}-y_{n}\right)\right)^{\mathbf{T}} B\left(\frac{y_{n}+y_{n+1}}{2}\right) \int_{0}^{1} \nabla H\left(y_{n}+\tau\left(y_{n+1}-y_{n}\right)\right) \mathrm{d} \tau\left(h+c \Delta \widehat{W}_{n}\right),
$$

which vanishes due to the fact that $C(y)$ is a Casimir.

3.3. Quadratic Hamiltonian functions. In this subsection, we show that the numerical integrator (2.1) is equivalent to the stochastic midpoint rule [17] in the case of quadratic Hamiltonian functions. Indeed, let us consider the following 
Hamiltonian function $H(y)=\frac{1}{2} y^{\mathbf{T}} D y+e^{\mathbf{T}} y$ with a constant symmetric matrix $D$ and a constant vector $e$. In this case, the numerical method (2.1) reads

$$
\begin{aligned}
y_{n+1} & =y_{n}+B\left(\frac{y_{n}+y_{n+1}}{2}\right) \int_{0}^{1}\left(\left(y_{n}+\tau\left(y_{n+1}-y_{n}\right)\right)^{\mathbf{T}} D+e^{\mathbf{T}}\right) \mathrm{d} \tau\left(h+c \Delta \widehat{W}_{n}\right) \\
& =y_{n}+B\left(\frac{y_{n}+y_{n+1}}{2}\right)\left(\frac{1}{2}\left(y_{n+1}+y_{n}\right)^{\mathbf{T}} D+e^{\mathbf{T}}\right)\left(h+c \Delta \widehat{W}_{n}\right),
\end{aligned}
$$

which is the stochastic midpoint rule applied to problem (1.1) with the above quadratic Hamiltonian function.

3.4. Order of the methods. The goal of this subsection is to prove that the scheme (2.1) has mean-square order 1 (see theorems 3.3 and 3.4 below). To do so, we first prove in Lemma 3.2 estimates on the remainder of an asymptotic expansion of our scheme (2.1), in order to compare it with the stochastic midpoint method from [17]. This order property of the energy-preserving scheme (2.1) is not directly linked to the geometric structure of the scheme. Hence, we simply denote by $f(y)=B(y) \nabla H(y)$ the Poisson vector field. We also set $g(y)=\nabla H(y)$. For the sake of simplicity of the presentation, we firstly restrict ourselves to the case where the Hamiltonian $H(y)$ and the structure matrix $B(y)$ are smooth functions of $y$ with globally bounded derivatives up to order 3 for $B$ and 4 for the function $H$. Thanks to the energy preservation of the scheme (see Proposition 3.1), this is indeed not a restriction for the applications we have in mind (see Section (4), as we explain in remarks 3.4 and 3.5 .

Let us recall the following.

Lemma 3.1. For all $p \in[1,+\infty)$, there exists a positive constant $C_{p}>0$ such that, for all $n \in \mathbb{N}$ and all $h \in(0,1)$, we have

$$
\left(\mathbb{E}\left(\left|\Delta W_{n}\right|^{p}\right)\right)^{1 / p} \leq C_{p} h^{1 / 2} \text { and }\left(\mathbb{E}\left(\left|\Delta \widehat{W}_{n}\right|^{p}\right)\right)^{1 / p} \leq C_{p} h^{1 / 2}
$$

Proof. Because $\Delta W_{n}$ is a real normal random variable with density $e^{-x^{2} / 2 h} / \sqrt{2 \pi h}$, we have, for some positive constant $C_{p}>0$,

$$
\mathbb{E}\left[\left|\Delta W_{n}\right|^{p}\right] \leq C_{p} h^{\frac{p}{2}} .
$$

Because $\left|\Delta \widehat{W}_{n}\right| \leq\left|\Delta W_{n}\right|$ almost surely, we infer that $\mathbb{E}\left[\left|\Delta \widehat{W}_{n}\right|^{p}\right] \leq C_{p} h^{\frac{p}{2}}$.

We are now able to prove the following.

LEMma 3.2. The numerical solutions provided by the numerical method (2.1) have the following asymptotic expansion 1 :

$$
y_{n+1}-y_{n}=a\left(y_{n}\right)\left(h+c \Delta \widehat{W}_{n}\right)+b\left(y_{n}\right)\left(h+c \Delta \widehat{W}_{n}\right)^{2}+c\left(y_{n}\right)\left(h+c \Delta \widehat{W}_{n}\right)^{3}+R_{n},
$$

where $a\left(y_{n}\right)=(B \nabla H)\left(y_{n}\right), b\left(y_{n}\right)=(B \nabla H)^{\prime}\left(y_{n}\right)(B \nabla H)\left(y_{n}\right) / 2$, and $c\left(y_{n}\right)$ are independent of $y_{n+1}$, and $R_{n}$ depends on $y_{n+1}$ and satisfies the following estimates:

$$
\mathbb{E}\left[\left\|R_{n}\right\|\right]=\mathcal{O}\left(h^{2}\right) \quad \text { and } \quad\left(\mathbb{E}\left[\left\|R_{n}\right\|^{2}\right]\right)^{\frac{1}{2}}=\mathcal{O}\left(h^{2}\right) .
$$

\footnotetext{
${ }^{1}$ Note that, in view of Lemma 3.1 one can see that the terms in the expansion (3.1) are not properly ordered and some of them could be included in the remainder. However, we keep this writing for the expansion because it is more compact and it helps understanding the computational process of the expansion.
} 
REMARK 3.3. Note that, in view of the hypotheses on the functions $B$ and $H$, the functions $a, b$, and $c$ above are bounded almost surely along the numerical solution by a constant which does not depend on $h \in(0,1)$. In particular, for all $p \geq 1$, their moments of order $p$ are finite and bounded by a constant which does not depend on $h$.

Proof. We proceed by successive Taylor expansions of $y_{n+1}-y_{n}$ in order to justify the expansion (3.1), and in particular to show that $R_{n}$ is of the form $(h+$ $\left.c \Delta \widehat{W}_{n}\right)^{4} r_{n}$, where $r_{n}$ depends on $y_{n+1}$ and all the moments of $r_{n}$ are bounded by a constant which does not depend on $h$. The estimates (3.2) then follow by applying the Cauchy-Schwartz inequality. In this proof, $R$ will denote a random variable which may vary from one line to the other and with finite moments of order $p$ for all $p \geq 1$ bounded by a constant which does not depend on $h \in(0,1)$. We will repeatedly use the fact that if $R$ and $S$ are two such random variables with appropriate dimensions, then so are $R S$ and $\left(h+c \Delta \widehat{W}_{n}\right) R$.

Let us start with the expansion of order 1 . Because $B$ and $g$ are smooth functions, we have

$$
B\left(y_{n}+\frac{y_{n+1}-y_{n}}{2}\right)=B\left(y_{n}\right)+\frac{1}{2} \int_{0}^{1} B^{\prime}\left(y_{n}+\frac{s}{2}\left(y_{n+1}-y_{n}\right)\right)\left(y_{n+1}-y_{n}\right) \mathrm{d} s,
$$

and

$$
\int_{0}^{1} g\left(y_{n}+\tau\left(y_{n+1}-y_{n}\right)\right) \mathrm{d} \tau=g\left(y_{n}\right)+\int_{0}^{1} \int_{0}^{\tau} g^{\prime}\left(y_{n}+s\left(y_{n+1}-y_{n}\right)\right)\left(y_{n+1}-y_{n}\right) \mathrm{d} s \mathrm{~d} \tau .
$$

Using the definition of the scheme (2.1) and plugging the expression of $y_{n+1}-y_{n}$ in the right hand side of the relations above, we obtain

$$
B\left(y_{n}+\frac{y_{n+1}-y_{n}}{2}\right)=B\left(y_{n}\right)+\left(h+c \Delta \widehat{W}_{n}\right) R,
$$

and

$$
\int_{0}^{1} g\left(y_{n}+\tau\left(y_{n+1}-y_{n}\right)\right) \mathrm{d} \tau=g\left(y_{n}\right)+\left(h+c \Delta \widehat{W}_{n}\right) R
$$

Taking the product of the two expansions above yields

$$
y_{n+1}-y_{n}=\left(h+c \Delta \widehat{W}_{n}\right) B\left(y_{n}\right) g\left(y_{n}\right)+\left(h+c \Delta \widehat{W}_{n}\right)^{2} R .
$$

We can now repeat this procedure to obtain the expansion of order 2 . We first write, by Taylor expansions and use of the definition of the scheme (2.1),

$$
B\left(y_{n}+\frac{y_{n+1}-y_{n}}{2}\right)=B\left(y_{n}\right)+\frac{1}{2} B^{\prime}\left(y_{n}\right)\left(y_{n+1}-y_{n}\right)+\left(h+c \Delta \widehat{W}_{n}\right)^{2} R,
$$

and

$$
\int_{0}^{1} g\left(y_{n}+\tau\left(y_{n+1}-y_{n}\right)\right) \mathrm{d} \tau=g\left(y_{n}\right)+\frac{1}{2} g^{\prime}\left(y_{n}\right)\left(y_{n+1}-y_{n}\right)+\left(h+c \Delta \widehat{W}_{n}\right)^{2} R .
$$

Inserting the expansion (3.3) of order 1 into the expressions above and taking the product, we obtain the expansion of order 2 : 


$$
\begin{aligned}
y_{n+1}-y_{n}= & \left(h+c \Delta \widehat{W}_{n}\right) B\left(y_{n}\right) g\left(y_{n}\right) \\
& +\frac{\left(h+c \Delta \widehat{W}_{n}\right)^{2}}{2}(\underbrace{B^{\prime}\left(y_{n}\right)\left(B\left(y_{n}\right) g\left(y_{n}\right)\right) g\left(y_{n}\right)+B\left(y_{n}\right) g^{\prime}\left(y_{n}\right)\left(B\left(y_{n}\right) g\left(y_{n}\right)\right)}_{=(B g)^{\prime}\left(y_{n}\right)(B g)\left(y_{n}\right)}) \\
& +\left(h+c \Delta \widehat{W}_{n}\right)^{3} R .
\end{aligned}
$$

Another step of this procedure, expanding $B\left(y_{n}+\left(y_{n+1}-y_{n}\right) / 2\right)$ and $\int_{0}^{1} g\left(y_{n}+\right.$ $\left.\tau\left(y_{n+1}-y_{n}\right)\right) \mathrm{d} \tau$ around $y_{n}$ up to order 3 thanks to the smoothness of $B$ and $g=\nabla H$, using the definition of the scheme in the terms of order 3 and the Taylor expansion of order 2 in the other terms, and then taking the product, yields the expansion (3.1) with an explicit expression of $c\left(y_{n}\right)$ which does not depend on $y_{n+1}$ or $\Delta \widehat{W}_{n}$ and with $R_{n}=\left(h+c \Delta \widehat{W}_{n}\right)^{4} R$. The estimates (3.2) follow by the Cauchy-Schwartz inequality. $\square$

We can now compare the method (2.1) with the well-known stochastic midpoint method to prove the following theorem.

TheOREm 3.3. Assume that the Hamiltonian $H(y)$ and the structure matrix $B(y)$ are smooth functions of $y$ with globally bounded derivatives up to order 3 for $B$ and 4 for the function $H$. Then the energy-preserving scheme (2.1) has mean-square order of convergence 1 .

Proof. Recall that $f=B \nabla H$. Using the same expansion method as the one we used in the proof of Lemma 3.2, we find that the stochastic midpoint method defined by

$$
\tilde{y}_{n+1}-\tilde{y}_{n}=f\left(\left(\tilde{y}_{n}+\tilde{y}_{n+1}\right) / 2\right)\left(h+c \Delta \widehat{W}_{n}\right),
$$

has an asymptotic expansion similar to the one proposed in 16

$$
\tilde{y}_{n+1}-\tilde{y}_{n}=\left(h+c \Delta \widehat{W}_{n}\right) a\left(\tilde{y}_{n}\right)+\left(h+c \Delta \widehat{W}_{n}\right)^{2} b\left(\tilde{y}_{n}\right)+\left(h+c \Delta \widehat{W}_{n}\right)^{3} \tilde{c}\left(\tilde{y}_{n}\right)+\tilde{R}_{n},
$$

with the same functions $a$ and $b$ as that of the expansion (3.1) of the scheme (2.1), with a function $\tilde{c}$ which does not depend on $\tilde{y}_{n+1}$ and a function $\tilde{R}_{n}$ of the form $\left(h+c \Delta \widehat{W}_{n}\right)^{4} R$, where $R$ is a function depending on $\tilde{y}_{n+1}$ through $\Delta \widehat{W}_{n}$ and with finite moments bounded by a constant which does not depend on $h \in(0,1)$. In view of the smoothness hypotheses on $B$ and $H$ and hence on $f$ and of the boundedness hypotheses on these functions and their derivatives, the random variables $a\left(\tilde{y}_{n}\right), b\left(\tilde{y}_{n}\right)$, and $\tilde{c}\left(\tilde{y}_{n}\right)$ are almost surely bounded by a constant which does not depend on $h$. Using the Cauchy-Schwarz inequality as in the proof of Lemma 3.2. yields

$$
\mathbb{E}\left[\left\|\tilde{R}_{n}\right\|\right]=\mathcal{O}\left(h^{2}\right) \quad \text { and } \quad\left(\mathbb{E}\left[\left\|\tilde{R}_{n}\right\|^{2}\right]\right)^{\frac{1}{2}}=\mathcal{O}\left(h^{2}\right) .
$$

Considering the difference between the solution $y_{n+1}$ provided by the energypreserving scheme (2.1) and the solution $\tilde{y}_{n+1}$ provided by the midpoint scheme (3.4) starting from the same point $y_{n}=\tilde{y}_{n}$, we obtain using (3.1) and (3.5)

$$
y_{n+1}-\tilde{y}_{n+1}=\left(c\left(y_{n}\right)-\tilde{c}\left(y_{n}\right)\right)\left(h+c \Delta \widehat{W}_{n}\right)^{3}+\left(R_{n}-\tilde{R}_{n}\right) .
$$

Because $R_{n}$ (see Lemma 3.2 ) and $\tilde{R}_{n}$ are of the form $\left(h+c \Delta \widehat{W}_{n}\right)^{4} R$, we have

$$
\left\|\mathbb{E}\left[R_{n}-\tilde{R}_{n}\right]\right\| \leq \mathbb{E}\left[\left\|R_{n}-\tilde{R}_{n}\right\|\right]=\mathcal{O}\left(h^{2}\right) .
$$


Moreover using the independence of the truncated Wiener increment $\Delta \widehat{W}_{n}$ with $y_{n}$, we have

$$
\mathbb{E}\left[\left(c\left(y_{n}\right)-\tilde{c}\left(y_{n}\right)\right)\left(h+c \Delta \widehat{W}_{n}\right)^{3}\right]=\mathbb{E}\left[c\left(y_{n}\right)-\tilde{c}\left(y_{n}\right)\right] \mathbb{E}\left[\left(h+c \Delta \widehat{W}_{n}\right)^{3}\right]=\mathcal{O}\left(h^{2}\right),
$$

because $\mathbb{E}\left[\left(h+c \Delta \widehat{W}_{n}\right)^{3}\right]=h^{3}+3 c^{2} h^{2}$. We infer that

$$
\mathbb{E}\left[y_{n+1}-\tilde{y}_{n+1}\right]=\mathcal{O}\left(h^{2}\right) .
$$

Moreover, using the triangle inequality in the right hand side of (3.6), the CauchySchwartz inequality and the estimation (3.7), we obtain

$$
\left(\mathbb{E}\left[\left\|y_{n+1}-\tilde{y}_{n+1}\right\|^{2}\right]\right)^{\frac{1}{2}} \leq \underbrace{\left(\mathbb{E}\left[\left\|c\left(y_{n}\right)-\tilde{c}\left(y_{n}\right)\right\|^{4}\right]\right)^{\frac{1}{4}}}_{<+\infty} \underbrace{\left.\mathbb{E}\left[\left(h+c \Delta \widehat{W}_{n}\right)^{12}\right]\right)^{\frac{1}{4}}}_{=\mathcal{O}\left(h^{3 / 2}\right)}+\underbrace{\left(\mathbb{E}\left[\left\|R_{n}-\tilde{R}_{n}\right\|^{2}\right]\right)^{\frac{1}{2}}}_{=\mathcal{O}\left(h^{2}\right)},
$$

which implies

$$
\left(\mathbb{E}\left[\left\|y_{n+1}-\tilde{y}_{n+1}\right\|^{2}\right]\right)^{\frac{1}{2}}=\mathcal{O}\left(h^{\frac{3}{2}}\right) .
$$

The stochastic midpoint method is known to have mean-square order 1 in the present context (see Theorem 2.6 in [17). Because the local difference between this scheme and the energy-preserving method (2.1) satisfies estimates (3.8) and (3.9), Lemma 2.1 of [16] with $p_{1}=2$ and $p_{2}=3 / 2$ ensures that the method (2.1) also has mean-square order 1 .

We can now relax the hypotheses on the boundedness of the functions $H(y)$ and $B(y)$ and their derivatives in the theorem above. First, the boundedness of $H$ is not mandatory (see Remark 3.4 below). Second, the boundedness of $f=B \nabla H$ and $B$ and their derivatives up to order 3 need not be global: local boundedness is sufficient to get a mean-square order 1 thanks to the energy preservation property (Proposition 3.1) of the scheme (2.1) (see Remark 3.5 below).

REMARK 3.4. The results above extend to several cases where $f=B \nabla H$ is smooth, sub-linear and all its derivatives are globally bounded. In fact, neither the results above nor the results of [16] require the boundedness of the function $H$ (they only need the boundedness of $g=\nabla H$ and its derivatives).

Remark 3.5. Because the method (2.1) exactly preserves the energy by Proposition 3.1, one can find, for problems such as those presented in Section 4, for all initial datum $y_{0}$, a convex subset of the phase space containing almost surely the numerical trajectories starting from $y_{0}$ on which the vector field $f$ and the function $B$ as well as their derivatives up to order 3 are bounded. Hence, the estimates in the proofs above extend to these cases straightforwardly. For example,

- for the harmonic oscillator starting at $y_{0}=\left(x_{0}, p_{0}\right) \in \mathbb{R}^{2}$ (see Section 4.1), such a convex set is the ball centered at the origin of the phase space with radius $\left(y_{0}^{2}+p_{0}^{2}\right) / 2$. Because $f$ is smooth on this compact set, it is bounded on it and so are its derivatives up to order 3.

- for the mathematical pendulum (see Section 4.3), such a convex set for an initial datum $y_{0}=\left(q_{0}, p_{0}\right)$ is for example $\{q \in \mathbb{R}\} \times\left\{p \in \mathbb{R} \quad|\quad| p \mid \leq \sqrt{p_{0}^{2}+4}\right\}$. Even if this set is not compact, the continuity and the $q$-periodicity of $f$ and its derivatives ensure that $f$ as well as its derivatives up to order 3 are bounded on such a convex set. 
- for the stochastic rigid body (see Section 4.2), for an initial datum $\left(y_{[1]}^{0}, y_{[2]}^{0}, y_{[3]}^{0}\right) \in \mathbb{R}^{3}$, a compact convex set on which the functions $f, B$, and their derivatives are bounded is the convex hull of the ellipsoid of equation $H\left(y_{[1]}, y_{[2]}, y_{[3]}\right)=H\left(y_{[1]}^{0}, y_{[2]}^{0}, y_{[3]}^{0}\right)$.

- for the Lotka-Volterra system (see Section 4.4), any numerical solution can be included in a rectangle which is the product of two compact intervals. Hence the functions $B$ and $f$ as well as their derivatives up to order 3 are bounded on such a compact (and convex) set.

We can summarise the results above as follows.

Theorem 3.4. Assume that the function $H$ and the matrix $B$ are of class $\mathcal{C}^{4}$, resp. $\mathcal{C}^{3}$, on $\mathbb{R}^{d}$ and that any energy-level $H(y)=$ Const is included in a convex set on which the functions $B$ and $\nabla H$ as well as all their derivatives up to order 3 are bounded. Then the scheme (2.1) applied to the stochastic problem (1.1) has mean-square order 1.

\section{Numerical experiments}

In this section, we perform some numerical experiments in order to illustrate the convergence order and various geometric properties of the proposed numerical integrators. We will compare the energy-preserving scheme (2.1) with the following numerical integrators:

- the classical Euler-Maruyama scheme of mean-square order 1/2 (applied to the converted Itō stochastic differential equation); see for example [11;

- the classical Milstein scheme of mean-square order 1 (applied to the converted Itō stochastic differential equation); see for example [11;

- the Euler-Heun scheme of mean-square order 1 introduced in [24]. When applied to Stratonovich differential equations

$$
\mathrm{d} y_{t}=a\left(y_{t}\right) \mathrm{d} t+\sigma\left(y_{t}\right) \circ \mathrm{d} W_{t},
$$

this numerical integrator reads

$$
\begin{aligned}
y_{n}^{\text {aux }} & =y_{n}+h a\left(y_{n}\right)+\sigma\left(y_{n}\right) \Delta \widehat{W}_{n} \\
y_{n+1} & =y_{n}+h a\left(y_{n}\right)+\frac{1}{2}\left(\sigma\left(y_{n}^{\text {aux }}\right)+\sigma\left(y_{n}\right)\right) \Delta \widehat{W}_{n} ;
\end{aligned}
$$

- the midpoint scheme of mean-square order 1 from [17. When applied to Stratonovich differential equations

$$
\mathrm{d} y_{t}=a\left(y_{t}\right) \mathrm{d} t+\sigma\left(y_{t}\right) \circ \mathrm{d} W_{t},
$$

this numerical method reads

$$
y_{n+1}=y_{n}+h a\left(\frac{y_{n}+y_{n+1}}{2}\right)+\sigma\left(\frac{y_{n}+y_{n+1}}{2}\right) \Delta \widehat{W}_{n} ;
$$

- the stochastic discrete gradient of mean-square order 1 from [10. When applied to Stratonovich differential equations, with a conserved quantity $I(y)$, of the form

$$
\mathrm{d} y_{t}=S\left(y_{t}\right) \nabla I\left(y_{t}\right) \mathrm{d} t+T\left(y_{t}\right) \nabla I\left(y_{t}\right) \circ \mathrm{d} W_{t},
$$


with two skew symmetric matrices $S(y)$ and $T(y)$, this numerical method reads

$$
y_{n+1}=y_{n}+h S\left(y_{n}\right) \bar{\nabla} I\left(y_{n}, y_{n+1}\right)+T\left(\frac{y_{n}+y_{n+1}}{2}\right) \bar{\nabla} I\left(y_{n}, y_{n+1}\right) \Delta \widehat{W}_{n} .
$$

In our case (1.1), we will take $I(y)=H(y)$ and the natural choices $S(y)=$ $T(y)=B(y)\left(\right.$ or $S(y)=T(y)=J^{-1}$ for stochastic canonical Hamiltonian systems). Furthermore,

$$
\bar{\nabla} I(y, \bar{y})=\frac{1}{2}\left(\bar{\nabla}_{1} I(y, \bar{y})+\bar{\nabla}_{1} I(\bar{y}, y)\right)
$$

is a symmetric discrete gradient (see [10] for more details) with

$$
\bar{\nabla}_{1} I(y, \bar{y}):=\left(\begin{array}{c}
\frac{I\left(\bar{y}_{[1]}, y_{[2]}, \ldots, y_{[d]}\right)-I\left(y_{[1]}, y_{[2]}, \ldots, y_{[d]}\right)}{\bar{y}_{[1]}-y_{[1]}} \\
\frac{I\left(\bar{y}_{[1]}, \bar{y}_{[2]}, \ldots, y_{[d]}\right)-I\left(\bar{y}_{[1]}, y_{[2]}, \ldots, y_{[d]}\right)}{\bar{y}_{[2]}-y_{[2]}} \\
\vdots \\
\frac{I\left(\bar{y}_{[1]}, \bar{y}_{[2]}, \ldots, \bar{y}_{[d]}\right)-I\left(\bar{y}_{[1]}, \bar{y}_{[2]}, \ldots, \bar{y}_{[d-1]}, y_{[d]}\right)}{\bar{y}_{[d]}-y_{[d]}}
\end{array}\right),
$$

where $y=\left(y_{[1]}, \ldots, y_{[d]}\right)^{\mathbf{T}}$ and $\bar{y}=\left(\bar{y}_{[1]}, \ldots, \bar{y}_{[d]}\right)^{\mathbf{T}}$.

4.1. The stochastic harmonic oscillator with one dimensional Wiener process. Let us first consider the stochastic harmonic oscillator 21]

$$
\mathrm{d}\left(\begin{array}{c}
x_{t} \\
p_{t}
\end{array}\right)=\left(\begin{array}{c}
p_{t} \\
-x_{t}
\end{array}\right)\left(\mathrm{d} t+c \circ \mathrm{d} W_{t}\right)
$$

where $c$ is a real non-negative parameter, $x_{t}, p_{t}$ are scalar stochastic processes, and $W_{t}$ is a one-dimensional Wiener process. The Hamiltonian thus reads $H(p, x)=p^{2} / 2+$ $x^{2} / 2$ and remains constant along the exact solution of the above problem. Noting $y=(p, x)^{\mathbf{T}}$, one thus gets equation (1.3). For this case, the scheme (2.2) reduces to

$$
\left(\begin{array}{l}
x_{n+1} \\
p_{n+1}
\end{array}\right)=\frac{1}{1+\frac{1}{4}\left(h+c \Delta \widehat{W}_{n}\right)^{2}}\left(\begin{array}{c}
\left(1-\frac{1}{4}\left(h+c \Delta \widehat{W}_{n}\right)^{2}\right) x_{n}+\left(h+c \Delta \widehat{W}_{n}\right) p_{n} \\
-\left(h+c \Delta \widehat{W}_{n}\right) x_{n}+\left(1-\frac{1}{4}\left(h+c \Delta \widehat{W}_{n}\right)^{2}\right) p_{n}
\end{array}\right)
$$

which is precisely the method proposed in [21, the stochastic midpoint scheme from [17, or the stochastic discrete gradient method from [10]. One can remark that, for this problem, one does not really need to use truncated random variables $\Delta \widehat{W}_{n}$ because the numerical method is well defined.

Figure 4.1 displays the numerical solutions in the phase space, the computed energies and the numerical position $x$ along one sample. For a better visibility in our figures, not all points of the numerical solutions are displayed. On the one hand, it can be observed that the numerical solutions given by the energy-preserving scheme remain on the initial energy circle so that this scheme has a long time stability. This stability can also be observed in the figure to the right, where the numerical positions of the energy-preserving method and Euler-Heun's method are displayed (the other numerical schemes offer similar behaviour as that of the Euler-Heun's method, the results are however not shown). On the other hand, one can observe 

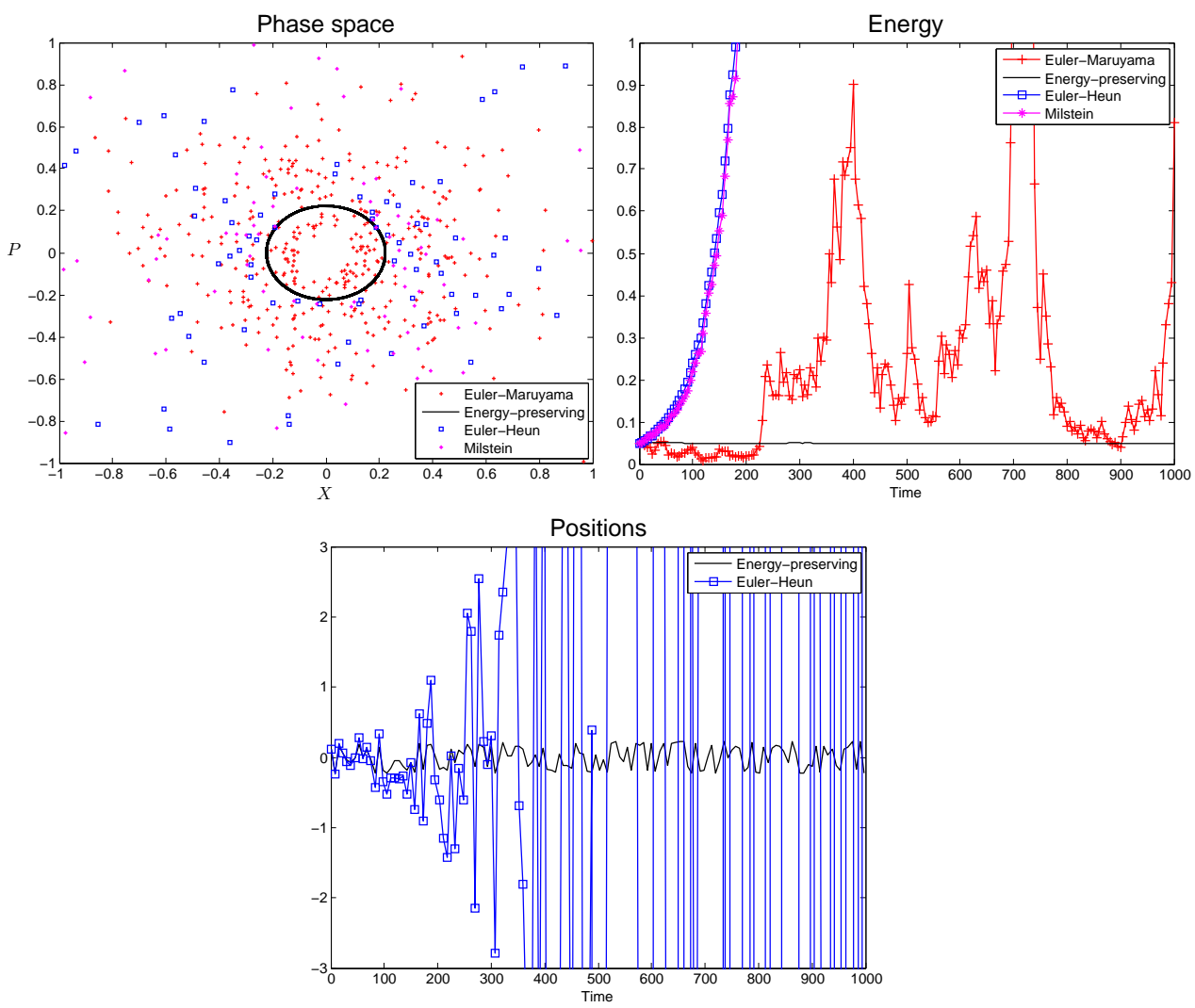

FIGURE 4.1. Stochastic harmonic oscillator with parameters $x_{0}=0.1, p_{0}=0.2$, and $c=1$. $N=10^{5}$ steps of length $h=10^{-2}$ for Euler-Maruyama (+), energy-preserving scheme (solid line), Milstein (*), and Euler-Heun ( $\square$ ).

that Euler-Maruyama's method, Euler-Heun's method, and Milstein's method are not appropriate for numerical simulations of the stochastic oscillator over long time intervals.

Figure 4.2 displays the mean-square errors at the final step

$$
\left(\mathbb{E}\left[\left\|y_{t_{N}}-y_{N}\right\|^{2}\right]\right)^{1 / 2}
$$

of the numerical integrators with the same parameters $x_{0}, p_{0}$, and $c$ as in the previous numerical experiment. Here, we take as the exact solution $y_{t_{N}}$ the numerical one using the Milstein scheme and a very small step size $h_{\text {exact }}=2^{-14}$. The expected values are approximated by computing averages over $M=5000$ samples. Convergence of order one, as stated by Theorem [3.4, is observed for the energy-preserving scheme. We remark that in this case the Hamiltonian of the problem is quadratic so that, by the result of Subsection 3.3. the energy-preserving scheme, the midpoint method, and the stochastic discrete gradient are the same numerical integrator.

4.2. A stochastic rigid body problem with one dimensional Wiener process. The equations of motion of a stochastic rigid body [14] are a Lie-Poisson 


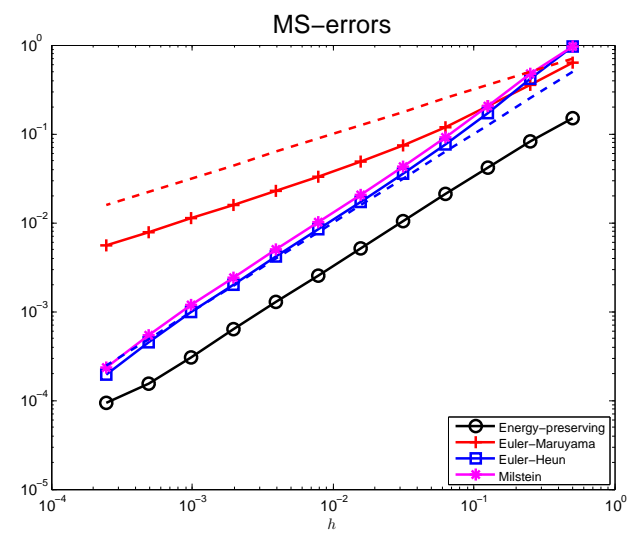

Figure 4.2. Stochastic harmonic oscillator. Plot of the order of convergence: mean-square errors at time $t_{\text {end }}=5 . M=5000$ samples used. The dashed lines have slopes $1 / 2$ and 1 .

system with Casimir $C(y)=\|y\|_{2}^{2}$ :

$$
\left(\begin{array}{l}
\mathrm{d} y_{[1]} \\
\mathrm{d} y_{[2]} \\
\mathrm{d} y_{[3]}
\end{array}\right)=\left(\begin{array}{ccc}
0 & -y_{[3]} & y_{[2]} \\
y_{[3]} & 0 & -y_{[1]} \\
-y_{[2]} & y_{[1]} & 0
\end{array}\right)\left(\begin{array}{l}
y_{[1]} / I_{1} \\
y_{[2]} / I_{2} \\
y_{[3]} / I_{3}
\end{array}\right)(\mathrm{d} t+c \circ \mathrm{d} W),
$$

where $y=\left(y_{[1]}, y_{[2]}, y_{[3]}\right)^{\mathbf{T}}$ and $I=\left(I_{1}, I_{2}, I_{3}\right)$ are the moments of inertia. The Hamiltonian

$$
H(y)=\frac{1}{2}\left(y_{[1]}^{2} / I_{1}+y_{[2]}^{2} / I_{2}+y_{[3]}^{2} / I_{3}\right)
$$

is thus a conserved quantity and method (2.1) exactly preserves it together with the quadratic Casimir

$$
C(y)=\frac{1}{2}\left(y_{[1]}^{2}+y_{[2]}^{2}+y_{[3]}^{2}\right) .
$$

Figure 4.3 (left) displays the computed Casimirs along one sample of the EulerMaruyama, the Euler-Heun, the Milstein, the stochastic discrete gradient, and the energy-preserving schemes. We used $N=300$ steps of the integrators with stepsize $h=10^{-2}$. It can be observed that the Casimir does not remain constant along the numerical solutions of the Euler-Maruyama, the Euler-Heun, the Milstein, and the stochastic discrete gradient methods. Similar behaviours are observed for the Hamiltonian (except for the stochastic discrete gradient method, which of course preserves this invariant). For the parameters in the problem, we used $c=1$, initial values $y_{0}=(0.8,0.6,0)$, and moments of inertia $I=(0.345,0.653,1)$. The mean-square orders of convergence at time $t_{\mathrm{end}}=1$ of the methods are also presented. We remark that, in this case too, the Hamiltonian of the problem is quadratic, so that the energypreserving scheme is the midpoint method.

\subsection{The mathematical pendulum with two dimensional Wiener pro-} cesses. Let us now consider a problem with a non-quadratic Hamiltonian, for example a stochastic perturbation of a mathematical pendulum

$$
\mathrm{d}\left(\begin{array}{c}
p_{t} \\
q_{t}
\end{array}\right)=\left(\begin{array}{c}
-\sin \left(q_{t}\right) \\
p_{t}
\end{array}\right)\left(\mathrm{d} t+c_{1} \circ \mathrm{d} W_{t}^{1}+c_{2} \circ \mathrm{d} W_{t}^{2}\right)
$$



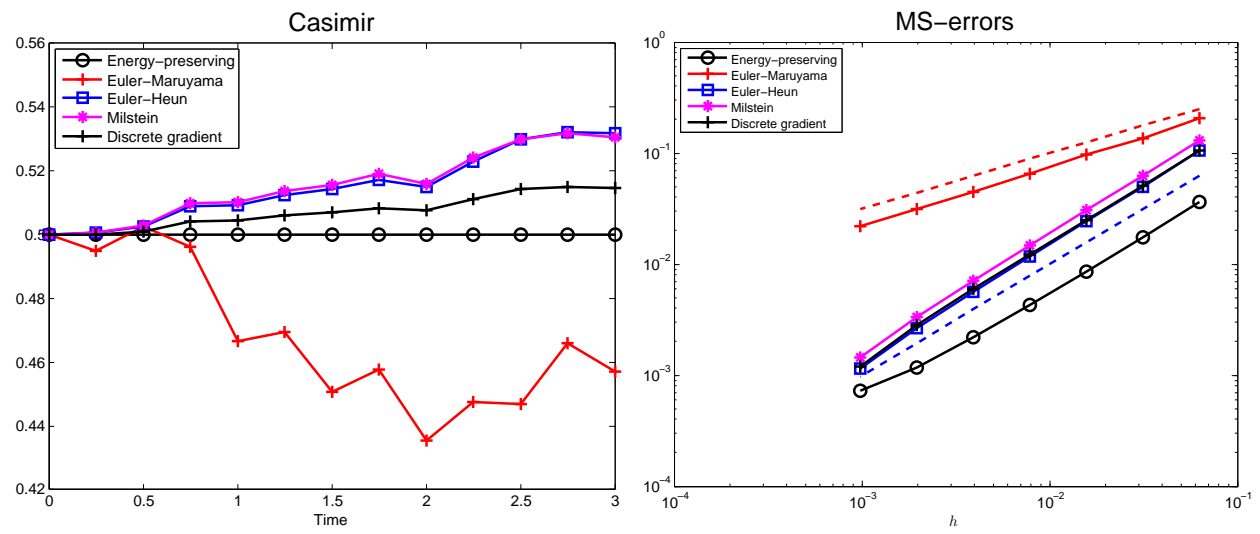

Figure 4.3. Computed Casimirs along the numerical solutions of a stochastic rigid body (left). The right plot displays the mean-square order of the schemes: the reference solution is computed using Milstein's method with $h_{\text {exact }}=2^{-12}$ and $M=1000$ samples are used for the expectations. The dashed lines have slopes $1 / 2$ and 1.

with two independent Wiener processes $W_{t}^{1}$ and $W_{t}^{2}$ and two real non-negative parameters $c_{1}, c_{2}$.

Figure 4.4 (top left plot) displays the energy $H(p, q)=\frac{1}{2} p^{2}-\cos (q)$ along one sample of the numerical solutions given by all the methods. The long time interval is $[0,500]$ and the numerical schemes use a step size $h=2^{-6}$. The energy does not remain constant along numerical solutions given by the Euler-Maruyama, the Euler-Heun, and the Milstein methods. However, the numerical solution given by the midpoint scheme remains almost constant over this long time interval as observed in this figure (top right plot). This behaviour is very interesting and could result from the symplecticity of this method. The energy along the stochastic discrete gradient method and the energy-preserving scheme is of course preserved. A good long time stability of the proposed scheme can be observed in this figure too (bottom plot), where the numerical positions of the energy-preserving method and Euler-Heun's method are displayed. A good long time stability of the stochastic discrete gradient and the midpoint methods is also observed, the results are however not shown for a better visibility.

We also show the convergence order of all the numerical integrators in figure 4.5 , Here, the reference solution is computed using the stochastic midpoint scheme with step size $h_{\text {exact }}=2^{-12}$ and $M=1000$ samples are used for the expectations. A meansquare order of convergence one is observed for the energy-preserving scheme.

4.4. Lotka-Volterra system. We finally consider a stochastic extension of the Lotka-Volterra system [6], for which

$$
B(y)=\left(\begin{array}{ccc}
0 & d y_{[1]} y_{[2]} & b d y_{[1]} y_{[3]} \\
-d y_{[1]} y_{[2]} & 0 & -y_{[2]} y_{[3]} \\
-b d y_{[1]} y_{[3]} & y_{[2]} y_{[3]} & 0
\end{array}\right),
$$

and

$$
H(y)=a b y_{[1]}+y_{[2]}-a y_{[3]}+\nu \ln y_{[2]}-\mu \ln y_{[3]} .
$$



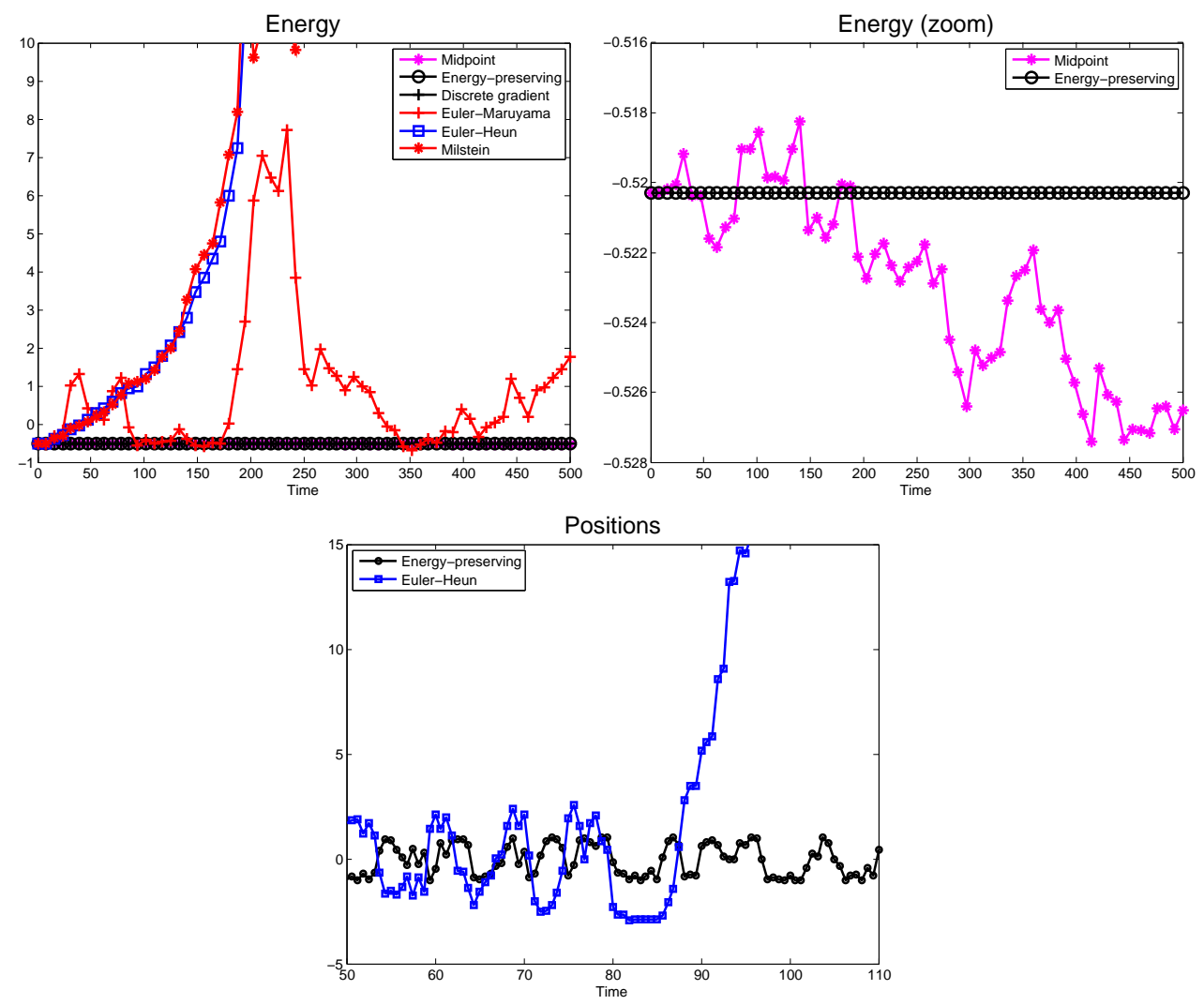

Figure 4.4. Stochastic mathematical pendulum with $p_{0}=0.2, q_{0}=1, c_{1}=1$, and $c_{2}=0.5$. Energy along all the numerical solutions (top left). Zoom for the computed energy of the midpoint and energy-preserving schemes (top right). Numerical positions of the energy-preserving scheme and Euler-Heun's method (bottom).

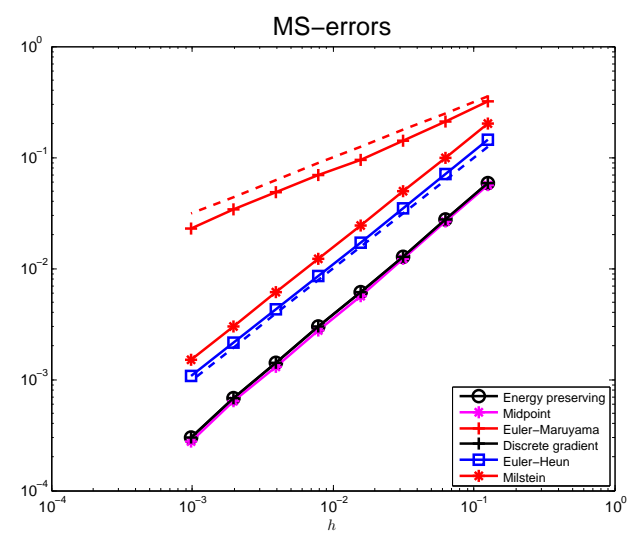

FIGURE 4.5. Stochastic mathematical pendulum. Mean-square errors at time $t_{\text {end }}=2: M=1000$ samples used and reference solution computed with the midpoint scheme and a step size $h_{\text {exact }}=2^{-12}$. The dashed lines have slopes $1 / 2$ and 1 . 

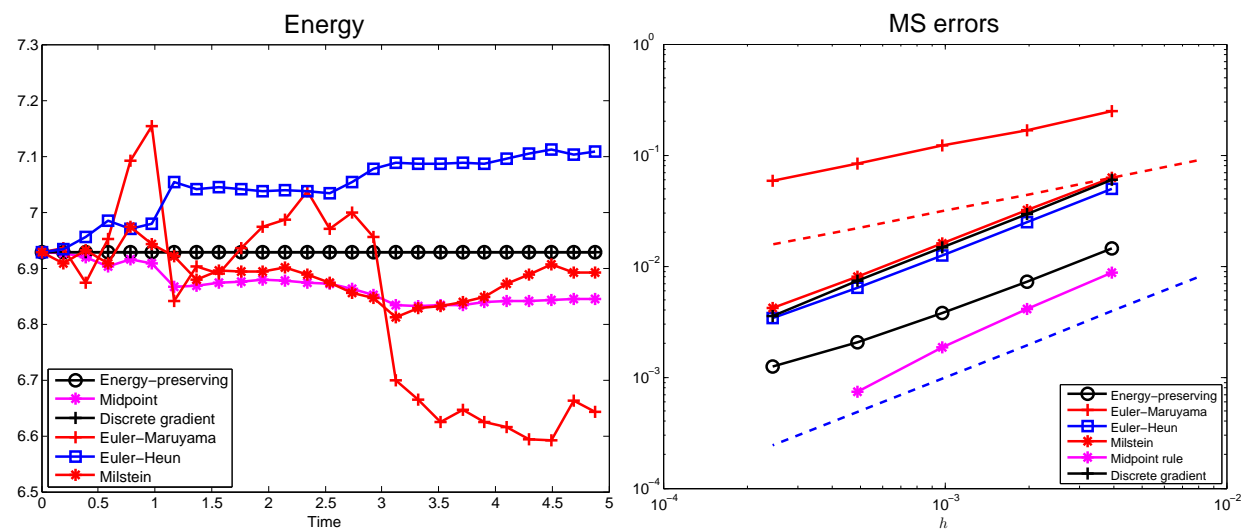

Figure 4.6. Hamiltonian along the numerical solutions of a Lotka-Volterra system (left plot). Loglog plot of the mean-square errors at time $t_{\text {end }}=1$ using $M=1000$ samples (right plot). The dashed lines have slopes $1 / 2$ and 1 .

For our numerical experiments, we choose the following parameters $a=-2, b=-1$, $d=-0.5, \nu=1, \mu=2$, and initial values $y_{0}=(1.0,1.9,0.5)$. The constant in front of the noise term is taken to be $c=0.5$. Figure 4.6 displays the Hamiltonian along one sample of the numerical solutions given by all the numerical integrators. We used the schemes with a step size $h=2^{-8}$ on the interval $[0,5]$. As expected, the total energy of our problem remains constant along the numerical solutions given by the energypreserving scheme (2.1) and the stochastic discrete gradient from [10] up to roundoff errors. This fact is not observed along numerical solutions given by the other schemes. In the convergence plot, a rate of convergence in the mean-square sense of one is observed for the energy-preserving method. For these numerical experiments, the reference solution was computed using the midpoint rule and a stepsize $h_{\text {exact }}=2^{-12}$.

\section{Conclusion and prospects}

In this paper, we have extended deterministic energy-preserving schemes from [6] to the case of randomised Poisson systems. We have proved that these numerical integrators are of mean-square order one, and preserve the energy and quadratic Casimirs for problem (1.1). A few questions remain open at the moment:

- What is the weak order of convergence of the proposed numerical schemes? We suspect that the weak order of the proposed scheme is the same as the weak order of the midpoint method from [17. This question will be addressed in a forthcoming work.

- Is it possible, as in the deterministic case, to raise the order of the energypreserving schemes? This seems a difficult task for the mean-square order. However, the techniques developed in [1] may be used to construct high weak order based on the energy-preserving scheme 2.1.

- In the end of Section 1 we addressed the question of developing numerical schemes that preserve more than one first integrals of the problem. This certainly deserves further theoretical investigations.

Acknowledgments. The authors would like to thank the anonymous referees for their valuable comments on the first version of this paper, Y. Komori for interesting discussions, and Inria, the institute of mathematics of the University of Basel and 


\section{ENERGY-PRESERVING INTEGRATORS FOR STOCHASTIC POISSON SYSTEMS}

UMIT Research Lab at Umeå University for their hospitality and financial support.

\section{REFERENCES}

[1] A. Abdulle, D. Cohen, G. Vilmart, and K.C. Zygalakis, High order weak methods for stochastic differential equations based on modified equations, SIAM J. Sci. Comput., 34(3), A1800A1823, 2012.

[2] J.M. Bismut, Mécanique Aléatoire, Lecture Notes in Mathematics, Springer-Verlag, Berlin, 866, 1981.

[3] N. Bou-Rabee and H. Owhadi, Stochastic variational integrators, IMA J. Numer. Anal., 29(2), 421-443, 2009.

[4] K. Burrage and P.M. Burrage, Low rank Runge-Kutta methods, symplecticity and stochastic Hamiltonian problems with additive noise, J. Comput. Appl. Math., 236(16), 3920-3930, 2012.

[5] E. Celledoni, R.I. McLachlan, D.I. McLaren, B. Owren, G.R.W. Quispel, and W.M. Wright, Energy-preserving Runge-Kutta methods, M2AN Math. Model. Numer. Anal., 43(4), 645$649,2009$.

[6] D. Cohen and E. Hairer, Linear energy-preserving integrators for Poisson systems, BIT, 51(1), 91-101, 2011

[7] E. Faou and T. Lelièvre, Conservative stochastic differential equations: Mathematical and numerical analysis, Math. Comput., 78(268), 2047-2074, 2009.

[8] O. Gonzalez, Time integration and discrete Hamiltonian systems, J. Nonlinear Sci., 6(5), 449467, 1996.

[9] E. Hairer, Energy-preserving variant of collocation methods, JNAIAM. J. Numer. Anal. Ind. Appl. Math., 5(1-2), 73-84, 2011.

[10] J. Hong, S. Zhai, and J. Zhang, Discrete gradient approach to stochastic differential equations with a conserved quantity, SIAM J. Numer. Anal., 49(5), 2017-2038, 2011.

[11] P.E. Kloeden and E. Platen, Numerical Solution of Stochastic Differential Equations, Applications of Mathematics (New York), Springer-Verlag, Berlin, 23, 1992.

[12] J.A. Lázaro-Camí and J.P. Ortega, Stochastic Hamiltonian dynamical systems, Rep. Math. Phys., 61(1), 65-122, 2008.

[13] J.A. Lázaro-Camí and J.P. Ortega, Reduction, reconstruction, and skew-product decomposition of symmetric stochastic differential equations, Stoch. Dyn., 9(1), 1-46, 2009.

[14] M. Liao, Random motion of a rigid body, J. Theoret. Probab., 10(1), 201-211, 1997.

[15] Q. Ma, D. Ding, and X. Ding, Symplectic conditions and stochastic generating functions of stochastic Runge-Kutta methods for stochastic Hamiltonian systems with multiplicative noise, Appl. Math. Comput., 219(2), 635-643, 2012.

[16] G.N. Milstein, Y.M. Repin, and M.V. Tretyakov, Mean-square symplectic methods for Hamiltonian systems with multiplicative noise, WIAS preprint No. 670, 2001.

[17] G.N. Milstein, Y.M. Repin, and M.V. Tretyakov, Numerical methods for stochastic systems preserving symplectic structure, SIAM J. Numer. Anal., 40(4), 1583-1604 (electronic), 2002.

[18] T. Misawa, Conserved quantities and symmetry for stochastic dynamical systems, Phys. Lett. A, 195(3-4), 185-189, 1994.

[19] T. Misawa, New conserved quantities derived from symmetry for stochastic dynamical systems, J. Phys. A, 27(20), L777-L782, 1994.

[20] T. Misawa, Conserved quantities and symmetries related to stochastic dynamical systems, Ann. Inst. Statist. Math., 51(4), 779-802, 1999.

[21] T. Misawa, Energy conservative stochastic difference scheme for stochastic Hamilton dynamical systems, Japan J. Indust. Appl. Math., 17(1), 119-128, 2000.

[22] T. Misawa, Symplectic integrators to stochastic Hamiltonian dynamical systems derived from composition methods, Math. Probl. Eng., Art. ID 384937, 12, 2010.

[23] E. Moro and H. Schurz, Boundary preserving semianalytic numerical algorithms for stochastic differential equations, SIAM J. Sci. Comput., 29(4), 1525-1549 (electronic), 2007.

[24] E. Platen, Zur Zeitdiskreten Approximation von Itoprozessen, PhD thesis, Diss. B. Imath. Akad. des Wiss. der DDR, Berlin, 1984.

[25] G.R.W. Quispel and D.I. McLaren, A new class of energy-preserving numerical integration methods, J. Phys. A, 41(4), 045206, 7, 2008.

[26] G. Unal, Symmetries of Itō and Stratonovich dynamical systems and their conserved quantities, Nonlinear Dynam., 32(4), 417-426, 2003.

[27] L. Wang, J. Hong, R. Scherer, and F. Bai, Dynamics and variational integrators of stochastic 
Hamiltonian systems, Int. J. Numer. Anal. Model., 6(4), 586-602, 2009.

[28] L.J. Wang, Variational Integrators and Generating Functions for Stochastic Hamiltonian Systems, PhD thesis, Karlsruhe Institute of Technology, 2007. 